\title{
Intervenciones de enfermería para la conservación de la función renal residual en diálisis peritoneal
}

\author{
Jesús Lucas Martín Espejo. DUE* - Antonia Concepción Gómez Castilla. DUE** - Maria de los Ángeles \\ Guerrero Rísco. DM*
}

Hospital Universitario Virgen del Rocío. Sevilla* Hospital Universitario Virgen Macarena. Sevilla**

\section{Resumen}

La conservación de la función renal residual es un factor importante a tener en cuenta en los pacientes en diálisis. En este trabajo nos hemos planteado los siguientes objetivos: estudiar los factores que influyen en el deterioro de la función renal residual de nuestros pacientes en DP. Definir los Diagnósticos de Enfermería y los Problemas de Colaboración en los que nuestras intervenciones contribuyen al enlentecimiento de este proceso.

La muestra estaba formada por 67 pacientes, con diuresis superior a $300 \mathrm{ml} /$ día al inicio en DP. Se observó la evolución de la función renal residual y su relación con la cantidad de glucosa y volumen semanal de líquido peritoneal utilizado, uso de icodextrina, técnica utilizada y otras variables relacionadas.

Seleccionamos los diagnósticos de enfermería (Perfusión tisular inefectiva-renal, Manejo inefectivo del régimen terapéutico, Riesgo de desequilibrio del volumen de líquidos, Riesgo de desequilibrio nutricional por defecto y exceso),

Correspondencia:

Jesús Lucas Martín Espejo

Plaza del Martinete 3, 4-I

41007 Sevilla

jlucasmartin@hotmail.com criterios de resultados e intervenciones con las actividades aplicadas de las taxonomías NANDA, NIC, NOC interrelacionadas, así como los problemas de colaboración.

En los resultados obtenidos, la glucosa y volumen de líquido peritoneal semanal eran inferiores en los pacientes que mantenían función renal residual. No encontramos diferencias en la función renal residual según la técnica de DP o el uso de icodextrina.

Los pacientes que conservan diuresis utilizan menos volúmenes y glucosa semanal, los parámetros nutricionales son mejores, no encontramos diferencia en el mantenimiento de la función renal residual según tipo de técnica, entre pacientes diabéticos y no diabéticos. Las intervenciones de Enfermería sobre los diagnósticos relacionados con desequilibrio en el volumen de líquido que consigan la normalización del peso, tensión arterial, ausencia de edemas, uso adecuado de glucosa en el dializado, etc. pueden favorecer el mantenimiento de la función renal residual.

PALABRAS CLAVE:

- DIÁLISIS PERITONEAL

- INTERVENCIONES DE ENFERMERÍA

- FUNCIÓN RENAL RESIDUAL

- ESTADO DE NUTRICIÓN 


\section{Nursing intervention for the conservation of residual kidney function in peritoneal dialysis}

\section{Abstrac}

The conservation of residual kidney function is an important factor to take into account in patients undergoing dialysis. In this work we have set the following goals: to study the factors that influence the deterioration of residual kidney function in our PD patients, to define the Nursing Diagnostics and Collaboration Problems in which our interventions contribute to slowing down this process.

The sample was made up of 67 patients, with diuresis in excess of $300 \mathrm{ml} /$ day at the beginning in PD. The evolution of the residual kidney function and its relationship with the quantity of glucose and weekly volume of peritoneal liquid used, use of icodextrine, technique used and other related variables were observed.

We selected the nursing diagnostics (Kidney-ineffective tissular perfusion, Ineffective handling of therapeutic regime, Risk of imbalance in volume of liquids, Risk of nutritional imbalance by deficiency or excess), outcomes and intervention criteria with the activities applied of interrelated NANDA, NIC and NOC taxonomies, and collaboration problems.

In the results obtained, the glucose and volume of weekly peritoneal liquid were lower in patients who maintained residual kidney function. We did not find differences in the residual kidney function according to the PD technique or use of icodextrine.

Patients who conserve diuresis use lower weekly volumes and glucose, nutritional parameters are better, we did not find any different in the maintenance of the residual kidney function depending on the type of technique, between diabetic and nondiabetic patients. Nursing interventions on diagnostics related to imbalance in the volume of liquid that achieve normal weight, blood pressure, absence of oedemas, adequate use of glucose in the dialysis patient, etc. can favour maintenance of residual kidney function.

\section{KEY WORDS:}

- PERITONEAL DIALYSIS

- NURSING INTERVENTIONS

- RESIDUAL KIDNEY FUNCTION

- STATE OF NUTRITION

\section{Introducción}

La conservación de la función renal residual (FRR) es un factor importante a tener en cuenta en los pacientes en diálisis. La preservación de parte de la función excretora renal de moléculas grandes, medias y pequeñas favorece un mejor equilibrio de electrolitos y ácido-base, proporcionando al paciente una disminución del tratamiento dialítico y un mejor estado general, a la vez que colabora en mantener funciones endocrinas que benefician al paciente en el control de la renina, anemia, metabolismo de la vitamina $D$ o del calcio y fósforo, lo que se traduce en más fuerza, mejor apetito (evitando así la malnutrición), menos dolores óseos, mejores parámetros nutricionales, menores restricciones de líquidos, etc., en conclusión, en una mejor calidad de vida ${ }^{1-3}$.

Algunos autores han confirmado que con la DP se conserva durante más tiempo la FRR, sobre todo en las técnicas continuas, Diálisis Peritoneal Continua Ambulatoria (DPCA) y Diálisis Peritoneal Automática (DPA) con día húmedo, y en los pacientes que usan icodextrina (Ico) durante los primeros meses de tratamiento. Estas modalidades de diálisis son mejor toleradas hemodinamicamente, ya que se producen menos episodios de hipotensiones y menos cambios bruscos de volemia que en HD, y, por lo tanto, menos isquemia renal. Además, en DP existe un grado de sobrehidratación en la mayoría de los pacientes, que favorece menores variaciones hemodinámicas, asimismo, la mejor biocompatibilidad de los líquidos y membrana usada en DP (membrana peritoneal) respecto a los de $H D^{1-6}$.

Otra explicación especulativa sobre la isquemia glomerular en DP podría ser que la filtración glomerular se afecte por la exposición repetida del peritoneo a un liquido no biológico en altas dosis ${ }^{6}$. También hay que considerar otros factores, como la enfermedad de base, ya que algunos procesos conservan peor la FRR que otras, como la glomerulonefritis o la nefro- 
patía diabética. Asimismo, otras incidencias que se pueden dar en DP, como la ganancia excesiva de peso, las peritonitis, la hipercalcemia, el uso de ciertos antibióticos etc., pueden influir en el ritmo de deterioro de la $\mathrm{FRR}^{1,3,7,8}$.

La pérdida de FRR no solo debe ser compensada en términos de Kt/V Urea, a través del aclaramiento peritoneal, sino incrementada para mantener una adecuada ingesta proteica, ya que no son superponibles los aclaramientos renal y peritoneal. De cualquier forma, la pérdida de $1 \mathrm{ml}$ en la FRR disminuye entre $0,25-0,30$ el $\mathrm{Kt} / \mathrm{V}$ semanal, lo que influye negativamente en la ingesta proteica $2,10,11$.

La FRR es un factor predictivo independiente de la supervivencia de los pacientes en DP, incluso mejor que los aclaramientos peritoneales de pequeñas moléculas6. Además, tiene un gran impacto, como ya se ha comentado, en los parámetros nutricionales y de adecuación del tratamiento.

Por otra parte, es necesario diseñar un Plan de Cuidados adecuado para la atención a estos pacientes ${ }^{12-14}$. Los Diagnósticos Enfermeros y Problemas de Colaboración serán un instrumento imprescindible en la ejecución de dicho Plan.

En este trabajo nos hemos planteado los siguientes objetivos:

- Estudiar los factores que influyen en el deterioro de la FRR de nuestros pacientes en DP.

- Definir los Diagnósticos de Enfermería y los Problemas de Colaboración en los que nuestras intervenciones contribuyen al enlentecimiento de este proceso.

\section{MATERIAL Y MÉTODO}

Se realizó un estudio observacional retrospectivo que incluyó a todos los pacientes adultos de los dos hospitales de Sevilla (Hospital Virgen Macarena y Virgen del Rocío) que estaban incluidos en programa de DP desde enero de 2003 a junio de 2005.

La muestra estudiada la formaron 67 pacientes, 38 hombres (57\%) y 29 mujeres ( $43 \%$ ). Se estudiaron las siguientes variables: edad, sexo, enfermedad de base, diabetes, tipo de tratamiento utilizado, tiempo en diálisis, causas de salida, número de peritonitis, uso y volumen de Ico, talla, peso al comienzo y al final del estudio; determinaciones analíticas (basal y final): hematocrito ( $\mathrm{Hto})$, hemoglobina ( $\mathrm{Hb})$, urea, creatinina, albúmina, existencia de edemas, dosis de eritropoyetina (EPO) mensual e índice de masa corporal (IMC). Se recogieron datos cada 3 meses durante el tiempo que el paciente permaneció en el estudio de: diuresis, filtrado glomerular (FG), ingesta proteica (nPCR), Kt/V, volumen total de líquido peritoneal usado semanalmente y cantidad de glucosa usada por semana. Se excluyeron del estudio a todos aquellos pacientes procedentes de HD con diuresis inferior a $300 \mathrm{ml}$ al comienzo del tratamiento y aquellos con menos de 3 meses en DP.

La aplicación de la metodología enfermera y la estandarización de los cuidados en estos pacientes ha seguido las fases del proceso enfermero:

- Valoración inicial integral según patrones funcionales de M. Gordon para la identificación de las necesidades más prevalentes.

- Selección de los diagnósticos de enfermería, criterios de resultados e intervenciones con las actividades aplicadas de las taxonomías NANDA, NIC, NOC interrelacionadas, así como los problemas de colaboración o interdisciplinares recogidos en el Plan de Cuidados estandarizado aplicado de forma individualizada y evaluado de forma periódica por el enfermero referente ${ }^{11-13}$.

Las fuentes de datos utilizadas fueron: historia médica, el programa PD-Adequest ${ }^{\circledR}$ y los registros de Enfermería de cada paciente.

El estudio estadístico se efectuó con el programa SPSS.12. Se analizaron los datos con distintos test no paramétricos: $U$ de Mann-Withney para comparar variables cuantitativas no relacionadas en dos grupos, chi cuadrado para comparar proporciones y Kruskall-Wallis para comparar variables cuantitativas en tres o más grupos. Las correlaciones entre diversas variables se analizaron mediante correlación lineal utilizando el coeficiente Rho de Spearman. 


\section{RESULTADOS}

La muestra estaba compuesta por 67 pacientes, 38 hombre (57\%) y 29 mujeres ( $43 \%$ ) con una edad media de $58 \pm 18$ años (rango 20-93). Los diferentes tratamientos utilizados por los pacientes fueron: 30 pacientes en DPCA, 29 pacientes en DPA con día húmedo y 8 pacientes en DPA con día seco. El tiempo medio en diálisis fue de 23,7 714 meses (rango 5-84). Usaron icodextrina, 28 pacientes, con un volumen medio de $9,9 \pm 3,9$ litros por semana, la diuresis media fue de $1467 \pm 718 \mathrm{ml} / 24$ horas (rango 300-3200), el filtrado glomerular medio fue de $6,33 \pm 2,74 \mathrm{ml} / \mathrm{mi}$ (rango 1,47-12,98), el $\mathrm{Kt} / \mathrm{V}$ medio de $2,52 \pm 0,62$ y se registraron 35 episodios de peritonitis.

La patología de base causante de la IRC se distribuyó de la siguiente manera: 11 poliquistosis renal, 8 nefropatía vascular, 5 enfermedades sistémicas, 16 nefropatía diabética, 16 glomerulonefritis y 11 IRC no filiada. Del total, 18 pacientes eran diabéticos.

Las causas de salida del programa fueron: 14 pacientes por trasplante y 15 por fallecimiento; pasaron a HD 5 pacientes y continúan en DP 33 pacientes.

En la tabla 1 se exponen los datos clínicos más significativos en el periodo basal y final. En la tabla 2 se exponen el número y porcentaje de pacientes que han conservado una diuresis superior a $300 \mathrm{ml}$ en los diferentes periodos del estudio, el volumen de la diuresis mantenida y el $F G$.

\section{Glucosa y volumen de LP se- manal}

Mediante el test no paramétrico de la U de Mann-Witney se compararon en pacientes con y sin FRR, en los diferentes puntos de corte estudiados: $6,12,18,24$, 30 y 36 meses. Nuestros resultados son:

- En el mes 6, no había diferencias significativas

- En el mes 12, los pacientes que mantenían diuresis habían utilizado menos líquido y menos glucosa semanal a lo largo de todo el tiempo de tratamiento, si bien solo la glucosa semanal alcanzaba una diferencia estadísticamente significativa basal $(391 \pm 347$ vs $717 \pm 330 \mathrm{gr}) \mathrm{p}=0,01$ en el mes $6(470 \pm 476$ vs $1085 \pm 657 \mathrm{gr}) \mathrm{p}=0,01, y$ en el mes $12(556 \pm 580$ vs1145 \pm 785 gr) $p=0,02$.

- En el mes 18 no encontramos diferencias significativas.

- En el mes 24, en los pacientes que mantenían FRR, encontramos diferencia estadísticamente significativa en la glucosa semanal $(313 \pm 400$ vs $580 \pm 405$ gr) $p=0,04$.

- En el mes 30, los pacientes que mantenían FRR habían utilizado menos líquido y menos glucosa semanal a lo largo de todo el tiempo de tratamiento, si bien solo la glucosa semanal $(242 \pm 275$ vs $790 \pm 620 \mathrm{gr}) \mathrm{p}=0,01$ alcanzaba una diferencia estadísticamente significativa.

- En el mes 36, los pacientes que mantenían diuresis habían utilizado menos líquido y menos glucosa semanal a lo largo de todo el tiempo de tratamiento, si bien solo el volumen de líquido de diálisis semanal alcanzaba una diferencia estadísticamente significativa en el mes 18 $(62 \pm 30$ vs $113 \pm 25 \mathrm{l}) \mathrm{p}=0,02$, mes $36(74 \pm 31$ vs $138 \pm 13$ I) $p=0,02$.

\begin{tabular}{lccc} 
Variables $(\mathrm{n}=\mathbf{6 7})$ & Basal & Final & $\mathbf{p}$ \\
\hline $\mathbf{H t} \mathbf{t}^{\circ}(\%)$ & $36 \pm 4.25$ & $35,3 \pm 7.08$ & $\mathrm{~ns}$ \\
\hline $\mathbf{H b}(\mathrm{g} / \mathrm{l})$ & $13,2 \pm 6.01$ & $13,5 \pm 11.4$ & $\mathrm{~ns}$ \\
\hline Urea $(\mathrm{mg} / \mathrm{dl})$ & $142 \pm 54$ & $116 \pm 40,7$ & 0,001 \\
\hline Creatinina (mg/dl) & $7,04 \pm 2,09$ & $8,07 \pm 2,76$ & 0,001 \\
\hline Albúmina (gr. \%) & $3,5 \pm 0.56$ & $3,5 \pm 0,53$ & $\mathrm{~ns}$ \\
\hline Epo/mensual (U.I.) & $24343 \pm 14136$ & $30597 \pm 22002$ & 0,02 \\
\hline Peso (Kg) & $71,1 \pm 14,9(35-102)$ & $73,3 \pm 15(38-103)$ & 0,001 \\
\hline IMC-basal (Kg/m2) & $26,9 \pm 4,4$ & $27,7 \pm 4,08$ & 0,001 \\
\hline Edemas Si / No (n) & $27 / 40$ & $39 / 28$ & $\mathrm{~ns}$ \\
\hline
\end{tabular}

Tabla 1. Diferentes variables al comienzo y al final del estudio

\begin{tabular}{lcccccc} 
Mes & $\mathbf{6}^{\circ}$ & $\mathbf{1 2 ^ { \circ }}$ & $\mathbf{1 8}^{\circ}$ & $\mathbf{2 4 ^ { \circ }}$ & $\mathbf{3 0 ^ { \circ }}$ & $\mathbf{3 6}^{\circ}$ \\
\hline $\mathbf{n}(\%)$ & $60(89,6)$ & $45(97,8)$ & $36(90 \%)$ & $29(82.3 \%)$ & $17(70.8 \%)$ & $11(72.6 \%)$ \\
Diuresis 24 horas & $1439 \pm 851$ & $1428 \pm 807$ & $1191 \pm 726$ & $1207 \pm 642$ & $1250 \pm 937$ & $1227 \pm 984$ \\
(ml/día) & $(350-3500)$ & $(300-3250)$ & $(300-3250)$ & $(300-2300)$ & $(300-3150)$ & $(300-3800)$ \\
\hline F.G. & $5,6 \pm 3,4$ & $5,5 \pm 3,3$ & $4,8 \pm 2,3$ & $4,6 \pm 2,3$ & $4,2 \pm 2,2$ & $3,8 \pm 2,1$ \\
(ml/min) & $(0,73-13,6)$ & $(1,0-12,2)$ & $(0,94-11,6)$ & $(1-10,5)$ & $(1,5-9,1)$ & $(1,1-7,2)$ \\
\hline
\end{tabular}

Tabla 2. Pacientes con diuresis y filtrado glomerular a lo largo del estudio 


\section{Comparación pacientes con/sin Icodextrina}

Al comparar mediante el test de la U de Mann-Wittney para muestras independientes, el volumen de líquido semanal (litros) y la glucosa semanal (gramos) utilizados a lo largo de todo el tiempo en DP, no se encontraron diferencias significativas según se utilizara o no Icodextrina.

\section{Comparación según diabetes}

La proporción de pacientes con diuresis superior a $300 \mathrm{ml}$ fue superior entre los pacientes no diabéticos, si bien solo a los 36 meses encontramos diferencias estadísticamente significativa $(p=0,03)$. No encontramos diferencias al aplicar el Test de la $U$ de Mann-Withney, entre la glucosa, litros de líquido semanal, $F G$, diuresis y tiempo de tratamiento entre los pacientes fueran o no diabéticos a lo largo del estudio.

\section{Comparación según la enfermedad de base}

Los pacientes con glomerulonefritis tenían una diuresis y $F G$ superiores, si bien solo fue significativo a los 6 meses $(p=0,04)$. El pequeño número de pacientes que forman los otros grupos de enfermedades, no permite comparar la proporción de pacientes con y sin FRR.

\begin{tabular}{|c|c|c|c|c|c|}
\hline \multirow[t]{2}{*}{ Mes } & Técnica utilizada & Diuresis (cc) & $p$ & FG (ml/min) & $p$ \\
\hline & DPCA $n=30$ & $1205 \pm 610$ & & $5,76 \pm 2,71$ & \\
\hline \multirow[t]{3}{*}{ Basal } & DPA DH $n=29$ & $1649 \pm 735$ & 0,02 & $6,78 \pm 2,85$ & ns \\
\hline & DPA DS $n=8$ & $1793 \pm 773$ & & $6,8 \pm 2,37$ & \\
\hline & DPCA $n=30$ & $1178 \pm 683$ & & $5,3 \pm 2,73$ & \\
\hline \multirow[t]{3}{*}{$3^{\circ}$} & DPA DH $n=27$ & $1582 \pm 727$ & 0.03 & $6,2 \pm 2,99$ & ns \\
\hline & DPA DS $n=8$ & $1850 \pm 956$ & & $7,19 \pm 3,79$ & \\
\hline & DPCA $n=30$ & $1200 \pm 748$ & & $5,05 \pm 3,13$ & \\
\hline \multirow[t]{3}{*}{$6^{\circ}$} & DPA DH $n=24$ & $1551 \pm 952$ & ns & $5,99 \pm 3,91$ & ns \\
\hline & DPA DS $n=6$ & $1783 \pm 945$ & & $6,39 \pm 2,7$ & \\
\hline & DPCA $n=23$ & $1115 \pm 734$ & & $4,54 \pm 3,22$ & \\
\hline \multirow[t]{3}{*}{$12^{\circ}$} & DPA DH $n=24$ & $1195 \pm 930$ & ns & $4,94 \pm 3,66$ & ns \\
\hline & DPA DS $n=5$ & $1980 \pm 967$ & & $7,00 \pm 2,73$ & \\
\hline & DPA DH $n=17$ & $981 \pm 624$ & & $3,65 \pm 2,16$ & \\
\hline \multirow[t]{3}{*}{$24^{\circ}$} & DPA DH $n=14$ & $878 \pm 796$ & ns & $4,23 \pm 2,76$ & ns \\
\hline & DPA DS $n=5$ & $1420 \pm 770$ & & $5,42 \pm 2,86$ & \\
\hline & DPCA $n=3$ & $83 \pm 227$ & & $1,66 \pm 0,47$ & \\
\hline \multirow[t]{2}{*}{$36^{\circ}$} & DPA DH $n=9$ & $420 \pm 859$ & ns & $3,59 \pm 2,33$ & ns \\
\hline & DPA DS $n=2$ & $1100 \pm 1272$ & & $3,72 \pm 3,95$ & \\
\hline
\end{tabular}

Tabla 3. Comparación de diuresis y filtrado glomerular según técnica de diálisis

\section{Comparación según la técnica de diálisis emple- ada}

Cuando se ha comparado la FRR (Kruskal- Wallis), según las diferentes técnicas utilizadas en los distintos periodos analizados solo encontramos diferencias estadísticamente significativa en la diuresis basal $(p=0,02)$ y a los 3 meses $(p=0,03)$, manteniendo un mayor volumen de diuresis los pacientes de DPA con día seco (tabla 3).

\section{Correlación lineal entre diuresis y FG con diver- sas variables}

Se estudió la correlación con: tiempo de tratamiento, número de peritonitis, PCR, volumen de icodextrina, glucosa semanal y volumen de líquido peritoneal (LP) semanal en los distintos cortes (6, 12, 18, 24, 30 y 36 meses) obteniéndose los siguientes resultados:

- El nPCR en el mes 24 se correlacionó negativamente con el número de peritonitis $(p=0,04, r=-$ $0,35)$ y el volumen de líquido semanal $(p=0,009$, $r=-0,45$ ).

- En el corte del mes $6(n=60)$ :

o La diuresis se correlacionó con el tiempo de tratamiento $(p=0,03, r=0,23)$ y la glucosa semanal $(p=0,001 r=-0,44)$

0 EI FG se correlacionó igualmente con el tiempo de tratamiento $(p=0,02, r=0,29)$ y la glucosa semanal $(p=0,02, r=-0,30)$.

- En el corte del mes $12(n=52)$ :

- La diuresis se correlacionó con la glucosa semanal $(p=0,006, r=-0,38)$

- EI FG se correlacionó con el tiempo de tratamiento $(p=0,02, r=-0,30)$ y la glucosa semanal $(p=0,04, r=-028)$.

- En el mes $18(n=40)$ : la diuresis se correlacionó con la glucosa semanal $(p=0,03, r=-0,34)$.

- En el corte del mes $24(n=36)$ : la diuresis se correlacionó con la glucosa semanal $(p=0,01, r=-$ 0,41 ) y el $F G$ con el volumen de Ico utilizado a la semana ( $p=0,01, r=-0,39$ ).

- En el mes $30(n=24)$ : la diuresis se correlacionó con la glucosa semanal $(p=0,02, r=-0,46)$ y el $F G$ con el tiempo de tratamiento $(p=0,02, r=0,46)$.

- En el mes $36(n=14)$ : la diuresis se correlacionó con el tiempo de tratamiento $(p=0,001, r=0,75)$ y el volumen de Ico $(p=0,02, r=-0,32)$. 


\section{Plan de Cuidados}

Respecto a los cuidados integrales, aplicados de forma metodológical2,14 es preciso resaltar el plan de enseñanza individualizada que recibieron estos pacientes cuando fueron incluidos en el programa de DP contemplando entre otros los siguientes temas: manejo de los diferentes líquidos de diálisis, la dieta prescrita, los medicamentos, el proceso de la enfermedad, los cuidados del catéter, etc y evaluando de forma periódica los conocimientos sobre dichos temas con actividades de la valoración del nivel de conocimientos en cada momento, para detectar lagunas a los errores o necesidades de refuerzo para fomentar un dominio gradual de la situación. Pretendíamos conseguir la: Adaptación psicosocial: cambio de vida (NOC: 1305) y poder solucionar el frecuente Deterioro de la adaptación. En este proceso de enseñanza por parte del enfermero referente se realizaron intervenciones como: Facilitar el aprendizaje (5520), Enseñanza individual (5606) y Disminución de la ansiedad (5820), que son determinantes para que el paciente domine los contenido del programa de aprendizaje que deben realizarse ante el evidente diagnóstico enfermero Déficit de conocimientos.

Los criterios de resultados y las intervenciones puestas en marcha para solucionar, medir, controlar los cambios o detectar precozmente los problemas (diagnósticos enfermeros/problemas de colaboración) relacionados con la pérdida de la FRR dentro del Plan de Cuidados más destacados fueron:

\section{PERFUSIÓN TISULAR INEFECTIVA -RENAL}

Criterios NOC: equilibrio electrolítico y ácido-base. Indicadores: sodio, potasio, urea, creatinina, etc. Intervenciones NIC (2150): Terapia de diálisis peritoneal incluyendo monitorización y cuidados en la diálisis peritoneal (todas las actividades descritas en esta intervención tienen relación directa o indirecta con la FRR y los factores que influyen en su deterioro así como con la contribución de enfermería en retrasar su pérdida).

\section{MANEJO INEFECTIVO DEL RÉGIMEN TE- RAPÉUTICO \\ Criterios NOC: \\ - Conducta de cumplimiento (1601) \\ - Conocimiento: régimen terapéutico (1813)}

- Participación: decisiones sobre asistencia sanitaria (1606)

- Conducta de fomento de la salud (1602)

Indicadores:

- Modifica la pauta orientada por el profesional (160106)

- Descripción de las responsabilidades de los propios cuidados para el tratamiento actual (181302)

- Utiliza técnicas de resolución de problemas para conseguir resultados deseados (160608)

- Utiliza conductas para evitar los riesgos (160201)

Intervenciones NIC:

- Modificación de la conducta (4360)

- Seguimiento telefónico (4920)

- Escucha activa (8190)

- Establecimiento de objetivos comunes (4410)

\section{RIESGO DE DESEQUILIBRIO DEL VOLU-} MEN DE LÍQUIDOS

Criterios NOC:

- Detección del riesgo (1908)

- Control del riesgo (1902)

- Hidratación (0602)

Indicadores:

- Reconoce los signos/síntomas que indican riesgo (190801)

- Modifica el estilo de vida para reducir el riesgo (190208)

- Ausencia de edemas periféricos (060203)

- Ausencia de dificultad respiratoria (060207)

Intervenciones NIC:

- Manejo de líquidos/electrólitos (2080) (actividades como control de peso y evolución, ajustes de electrólitos en las bolsas, registro de balances, restricciones, evaluar signos de pérdidas, etc).

\section{RIESGO DE DESEQUILIBRIO NUTRICIO- NAL POR DEFECTO Y EXCESO \\ Criterios NOC:}

- Estado nutricional: ingestión de nutrientes (1909)

- Control de peso (1612)

Indicadores:

- Ingestión proteica (100902)

- Ingestión mineral (Ca, P, K, Na) (100906)

- Mantiene el peso óptimo (161222)

Intervenciones NIC:

- Manejo de la nutrición (1100)

- Facilitar la auto responsabilidad (4480) 


\section{RIESGO DE INFECCIÓN \\ Criterios NOC:}

- Control de riesgo (1902)

- Integridad del acceso de diálisis (catéter peritoneal) (1105)

\section{Indicadores:}

- Reconocer el riesgo (190201)

- Ausencia de supuración local (110503)

Intervenciones NIC:

- Control de infecciones (6540)

- Mantenimiento de dispositivo para diálisis (catéter peritoneal) (2440)

- Vigilancia de la piel (3590)

- Cuidado de las heridas (3660)

En cuanto a las complicaciones potenciales hemodinámicas y metabólicas en las que debemos actuar en la detección precoz de signos y síntomas, destacamos las siguientes intervenciones relacionadas con la pérdida de la FRR:

- Manejo de la hipovolemia (2380)

- Manejo de la hipervolemia (4170)

- Manejo de la hiperglucemia (2000)

- Manejo de la hipoglucemia (2130)

- Cuidados cardíacos (4040)

- Oxigenoterapia (3320)

- Manejo de la medicación (2380)

\section{Discusión}

En el grupo estudiado, un alto porcentaje de los pacientes conservaba un volumen de diuresis que hemos considerado significativa (superior a $300 \mathrm{ml}$ ) en todos los cortes (aunque va disminuyendo a lo largo de los meses), posiblemente porque la pérdida de la FRR se asocia con frecuencia a fallo de técnica por dosis de diálisis y/o ultrafiltración (UF) insuficientes, y obliga al paso del paciente a HD. Asimismo, también en nuestra serie, la pérdida de la FRR se asocia a mayor mortalidad6, que puede contribuir a que los pacientes que siguen vivos y en DP son los que mantienen FRR (en el presente trabajo había una correlación positiva entre la diuresis y el FG con el tiempo de tratamiento).

Los resultados obtenidos en nuestro estudio ponen de manifiesto que los pacientes que conservan una diuresis significativa utilizan menos glucosa semanal y menor volumen de líquido peritoneal a lo largo de su seguimiento. El uso excesivo de concentrados hipertónicos y de líquido peritoneal para conseguir UF elevadas se ha considerado una de las causas que acelera la pérdida de la $\mathrm{FRR}^{1-5}$. Sin embargo, en nuestro trabajo observamos que los pacientes con diuresis inferior parten de entrada de valores significativamente más bajos de diuresis y de $\mathrm{FG}$, por lo que el uso de mayor volumen de líquido peritoneal y glucosa no podría considerarse causa sino consecuencia de una menor FRR que obliga a un mayor aclaramiento peritoneal para compensar y conseguir una dosis de diálisis y UF adecuados.

Otra consecuencia del aumento de glucosa en el LP, que se asocia a su vez a una FRR inferior, es la disminución de la ingesta proteica, al aumentar el aporte energético por el LP, lo que disminuye el apetito ${ }^{9}$. Corroborando estos hechos, en nuestro trabajo hemos observado peores valores de ciertas variables relacionadas con la nutrición en los pacientes con diuresis inferior a $300 \mathrm{ml}$. como la albúmina, ingesta proteica medida por nPCR e IMC.

Un factor que se piensa puede ser importante en la perdida de FRR es la incidencia de peritonitis, que puede influir negativamente no solo por el posible efecto del estado inflamatorio, sino también por el tratamiento con antibióticos nefrotóxicos ${ }^{10}$. En nuestro estudio, la incidencia de peritonitis se correlacionó con el tiempo de tratamiento, como es de esperar, pero no con la diuresis, $F G$, ni volumen o concentración de glucosa en el LP; tampoco era distinto el número de peritonitis en los pacientes con o sin diuresis significativa, por lo que parece que no influyó en el mantenimiento de la FRR directa o indirectamente.

No hemos observado diferencias entre el grupo que utilizaba icodextrina y el que no, en cuanto al volumen de LP o glucosa empleada; sí se ha observado que en el grupo de pacientes con icodextrina había un mayor número de pacientes sin FRR, significativo en el mes 18 y con valores muy parecidos en los otros meses. Por lo que parece que, en nuestro grupo, la icodextrina ha influido poco en el mantenimiento de la FRR. En estudios previos se ha comunicado un efecto protector de esta sustancia sobre la FRR4. 
Nuestros resultados no concuerdan con los de otros autores ${ }^{1-3}$ que aconsejan las técnicas continuas como más favorecedora de la conservación de la FRR, ya que no hemos encontrado diferencias según la técnica utilizada a lo largo de la evolución en el porcentaje de pacientes con diuresis. Hemos de tener en cuenta que los pacientes con DPA y día seco partían de valores de diuresis y $F G$ superiores, que se van igualando a las otras técnicas a lo largo del seguimiento, posiblemente porque los pacientes que siguen en DP son los que mantienen superior FRR.

El uso de mayor volumen de líquido utilizado y las concentraciones más altas de glucosa que hemos encontrado en nuestro estudio en los pacientes que van perdiendo la diuresis, puede guardar relación con diagnósticos enfermeros detectados frecuentemente en nuestros pacientes como: "Riesgo de desequilibrio nutricional por exceso/defecto", "Desequilibrio de volumen de líquidos", "Deterioro de la eliminación urinaria", "Exceso de volumen de líquidos" y "Manejo inefectivo/efectivo del régimen terapéutico"1214, donde intervenciones de Enfermería como "Manejo de la nutrición", "Manejo de los líquidos y electrolitos" (ingesta de líquidos, sal y uso de las concentraciones del dializado), "Manejo de la diuresis y medicación", "Manejo del peso", "Modificaciones de la conducta", "Identificación de riesgos", "Enseñan$z^{\prime \prime}$ y "Seguimiento telefónico", son imprescindibles para evitar el uso inadecuado de las concentraciones de glucosa del dializado que pueda facilitar la isquemia glomerular y deteriorar de forma prematura la FRR.

En las evoluciones del plan de cuidados pudimos observar cómo los pacientes que antes perdieron la FRR habían tenido un retroceso en las escalas de los indicadores del plan de cuidados sobre todo en los relacionados con una modificación de la conducta o el estilo de vida enfocada a nuestras enseñanzas y recomendaciones.

En los procesos de empeoramiento del estado de salud de estos pacientes que precisan ingreso hospitalario: edema agudo de pulmón, peritonitis, angor, etc. conoceremos a través del cuaderno de continuidad de cuidados el grado de afectación de la FRR y de los diagnósticos relacionados así como del resto de los patrones funcionales que tendremos que revalorar para ayudar al paciente y cuidador a afrontar y manejar el nuevo régimen terapéutico.

Podemos concluir que, en nuestro estudio, los pacientes que conservan diuresis utilizan menos volúmenes y menos glucosa semanal, los parámetros nutricionales han sido mejores en paciente con diuresis, no hemos encontrado diferencia en el mantenimiento de la FRR según tipo de técnica y no hemos encontrado diferencias entre pacientes diabéticos y los que no lo son. En cuanto al segundo objetivo del estudio, las intervenciones de enfermería sobre los diagnósticos relacionados con desequilibrio en el volumen de líquido que consigan la normalización del peso y la tensión arterial, la ausencia de edemas, el uso adecuado de glucosa en el dializado, etc. pueden favorecer el mantenimiento de la FRR.

\section{Agradecimiento}

a Dña. Trinidad López Sánchez y D. Francisco Cirera Segura, por su colaboración en la elaboración de este trabajo.

\section{BIBLIOGRAFÍA}

1. Molina Núñez M. Conferencia "Abordaje multidisciplinar para el mantenimiento de la función renal residual". XXX Congreso de la SEDEN. 12-15 0ctubre 2005. Málaga.

2. Caravaca F, Cid MC, Galán J, García MC et al. Relación entre el aclaramiento de urea y la tasa del catabolismo proteico en pacientes de DPCA: importancia de la función renal residual. Nefrología 1996; 16 (3).

3. Valderrabanos F. Tratado de Hemodiálisis. BarceIona: Editorial Medica Jims SL; 1999.

4. Bajo MA, Olea $T$, del Peso G, Ros S, Castro MJ, Selga R. El uso de la Icodextrina desde el inicio de la Diálisis Peritoneal preserva mejor la función renal residual, pero no modifica el transporte peritoneal de agua y solutos. $V$ Reunión Nacional de Diálisis Peritoneal. Toledo 2005. p. 23.

5. Ramírez B, Pulido M, Roncal D. Lobo MT et al. Función renal residual. Su importancia en el manejo del paciente en diálisis. Rev Soc Esp Enferm Nefrol. 2001; Primer trimestre.13:68-71. 
6. Gallar P, Ortega 0, Gutiérrez M, Carreño A et al. Diálisis Peritoneal Automatizada (DPA): impacto sobre la función renal residual (FRR) y sobre la permeabilidad de la membrana peritoneal" Nefrología. 2001; 21(2):200-203.

7. Andréu Periz L, Force Sanmartín E. 500 cuestiones que plantea el cuidado del enfermo renal. Barcelona: Masson; 1997.

8. Borrego F, Selga R, de Álvaro F, Bajo MA et al. Seguimiento de la función renal residual en pacientes en DPCA. La influencia de los aclaramientos peritoneales y de los fármacos. Nefrología. 1993; 13(1):37-46

9. Sansone A. Cirugeda MA, Bajo G. del Peso et al. Actualización de protocolos en la practica clínica de Diálisis Peritoneal, año 2004. Nefrología. 2004; 24(5): 410-445.
10. Caravaca F, Arrobas M, Dominguez C. Influence of residual renal function on dietary protein and caloric intake in patients on incremental peritoneal dialysis. Perit Dial Int. 1999; 19(4):350-6.

11. Borrego F, Selga R, de Álvaro F, Bajo MA et al. Seguimiento de la función renal residual en pacientes en DPCA. La influencia de los aclaramientos peritoneales y de los fármacos. Nefrología. 1993; 13(1):37-46.

12. Diagnósticos Enfermeros: Definiciones y Clasificación 2003-2004 Nanda Internacional. Ediciones Elsevier; 2005.

13. Johnson M, Maas M. Clasificación de Resultados de Enfermería (CRE). $3^{a}$ edición. Harcourt; 2005.

14. McCloskey J. Clasificación de Intervenciones de Enfermería. $3^{a}$ edición. Harcourt; 2002. 